\title{
A Novel Phase Sensitive Amplifier Based QPSK Regenerator Without Active Phase- Locking
}

Kjøller, Niels-Kristian; Da Ros, Francesco; Røge, Kasper Meldgaard; Guan, Pengyu; Galili, Michael; Oxenløwe, Leif Katsuo

\section{Published in:}

Proceedings of IEEE Photonics Conference

Link to article, DOI:

10.1109/IPCon.2015.7323558

Publication date:

2015

Document Version

Peer reviewed version

Link back to DTU Orbit

Citation (APA):

Kjøller, N-K., Da Ros, F., Røge, K. M., Guan, P., Galili, M., \& Oxenløwe, L. K. (2015). A Novel Phase Sensitive Amplifier Based QPSK Regenerator Without Active Phase-Locking. In Proceedings of IEEE Photonics Conference IEEE. https://doi.org/10.1109/IPCon.2015.7323558

\section{General rights}

Copyright and moral rights for the publications made accessible in the public portal are retained by the authors and/or other copyright owners and it is a condition of accessing publications that users recognise and abide by the legal requirements associated with these rights.

- Users may download and print one copy of any publication from the public portal for the purpose of private study or research.

- You may not further distribute the material or use it for any profit-making activity or commercial gain

- You may freely distribute the URL identifying the publication in the public portal 


\title{
A Novel Phase Sensitive Amplifier Based QPSK Regenerator Without Active Phase-Locking
}

\author{
N. K. Kjøller, F. Da Ros, K. M. Røge, P. Guan, M. Galili, and L. K. Oxenløwe \\ DTU Fotonik, Technical University of Denmark. DK-2800 Kgs. Lyngby, Denmark.
}

\begin{abstract}
We propose a novel QPSK regenerator scheme based on phase sensitive amplification of a pre-conditioned signal avoiding active phase-locking. Signal pre-conditioning is demonstrated experimentally with error-free (BER $<10^{-9}$ ) performance for a 10-Gbaud QPSK signal.
\end{abstract}

Keywords-optical signal processing; cross phase modulation; phase sensitive amplification

\section{INTRODUCTION}

The increasing demand for capacity is driving optical communication systems towards higher order modulation formats with increased spectral efficiency, such as quadrature phase-shift keying (QPSK) and quadrature amplitude modulation (QAM). In recent years, phase sensitive amplifiers (PSAs) have been applied to alloptically regenerate binary phase shift keying (BPSK) [1], QPSK [2] and 8-QAM [3] signals. A requirement for such systems is a stable pump-signal phase relation at the PSA input. This has been achieved with active phase stabilization such as electronic feedback loops.

Recently, we proposed and demonstrated a novel scheme for PSA based regeneration of BPSK signals without active phase-locking by employing an optical signal pre-conditioning [4]. Here, we propose how to extend the regeneration scheme to QPSK signals and demonstrate preconditioning of a QPSK signal to achieve stable phase relative to a set of pumps suitable for QPSK regeneration. Preliminary investigation of PSA operation show error-free transfer of data modulation as well as the expected phase sensitive response, setting the stage for future demonstration of passively locked PSA based QPSK regenerators.

\section{BASIC PRINCIPLE}

The main idea behind the signal pre-conditioning is to locally generate the required set of phase-locked carriers to be used as signal and pumps in the PSA, and transfer the phase modulation of the incoming data to the signal carrier, without disturbing the overall phase relationship between the phase-locked waves. First, the incoming QPSK signal is passed through a delay interferometer (DI) to generate a four-level amplitude modulated signal. Next, the pumps are sent one way round a fiber loop while the intended signal carrier and the four-level amplitude modulated signal are sent the opposite way round. XPM between the copropagating waves takes place in a highly nonlinear fiber (HNLF) and the locally generated carrier thus receives QPSK modulation. All waves propagate through the same fiber in the loop configuration, thus the phase relation is maintained. The output is three phase-locked carriers, one of which now containing the phase modulated data. For use as a regenerator, the set is to be sent to a second HNLF where phase sensitive amplification can be employed to create a wavelength converted copy of the signal with reduced phase noise. As first demonstrated in [2], QPSK regeneration results from interference between four-wave mixing (FWM) generated idlers having the phase $3 \phi$ and $-\phi$, with $\phi(\mathrm{t})$ being the phase of the QPSK modulated signal carrier.

\section{EXPERIMENTAL SETUP}

The setup is illustrated in Fig. 1. An optical frequency comb with a line spacing of $40 \mathrm{GHz}$ is generated by phase modulating (PM) at a frequency of $40 \mathrm{GHz}$ a continuous wave (CW) signal at $1544 \mathrm{~nm}$ from a narrow linewidth $(<100 \mathrm{kHz})$ external cavity laser. Three phase-locked carriers - a signal (S) and two pumps (P1, P2) positioned $-80 \mathrm{GHz}$ and $+240 \mathrm{GHz}$ relative to $\mathrm{S}-$ are extracted using an optical processor (WSS). The three co-polarized carriers are aligned at $45^{\circ}$ with respect to the primary polarization axis of a polarization maintaining fiber (PMF) with a total birefringence matched to half the pump-pump spacing, rotating the polarization of $\mathrm{S} 90^{\circ}$ relative to $\mathrm{P} 1$ and $\mathrm{P} 2$. This allows using a polarizing beam splitter (PBS) to send $\mathrm{S}$ and $\mathrm{P} 1, \mathrm{P} 2$ in opposite directions round a loop containing 500-m HNLF.
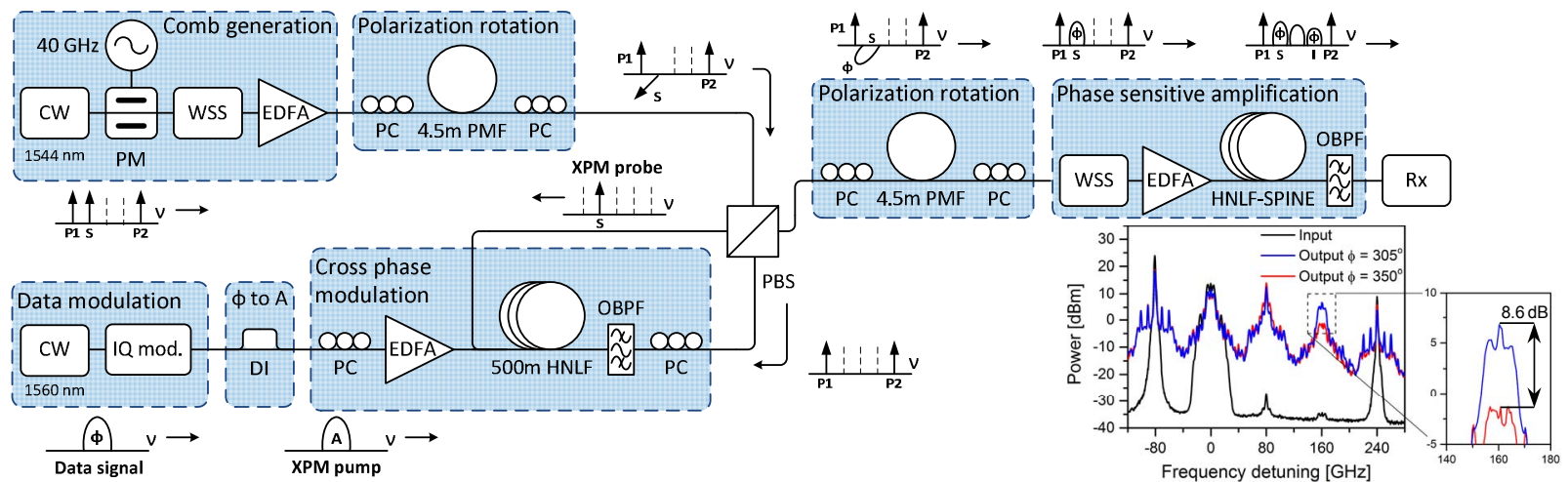

Fig. 1. Experimental setup for the PSA based QPSK regenerator without active phase-locking. Input and output spectra of the HNLF-SPINE are shown in inset. 
An NRZ QPSK data signal is generated by modulating a $\mathrm{CW}$ at $1560 \mathrm{~nm}$ (linewidth $<100 \mathrm{kHz}$ ) at 10 Gbaud using a standard IQ modulator, by applying a pseudorandom bit sequence (PRBS) of length $2^{f}-1$ and its logically inverted copy to the data inputs. The QPSK signal is converted to a four-level amplitude signal in a 1 bit (100 ps) fiber DI, with the delay carefully adjusted to ensure equal spacing between the four amplitude levels. The XPM pump is amplified using an erbium doped fiber amplifier (EDFA) and coupled into the loop in the same direction as S. By carefully adjusting the XPM pump power, the phase of $S$ is encoded with $0, \pi / 2, \pi$ or $3 \pi / 2$. After the HNLF, a $5-n m$ optical band pass filter (OBPF) is used to select only the three original phase-locked carriers. Using a polarization controller (PC) $\mathrm{P} 1, \mathrm{P} 2$ and the now QPSK modulated $\mathrm{S}$ are directed to the remaining port of the PBS. A second PMF identical to the first one is used to rotate the three carriers to the same plane of polarization.

A second WSS is used to remove out of band noise and adjust power and phase of $S$ relative to the pumps, in order to maximize the phase sensitive response. The signal $\mathrm{S}$ and pumps P1, P2 are then launched into a $250 \mathrm{~m}$ strained HNLF with stable phase-matching for improved nonlinear efficiency (HNLF-SPINE). After the HNLF, P1 and P2 are removed using a tunable flat-top OBPF and the output is sent to a pre-amplified receiver ( $\mathrm{Rx})$, comprising a 1 bit fiber DI followed by a balanced photodiode and an electrical low-pass filter with a cut-off at $7.5 \mathrm{GHz}$, for bit-error-ratio (BER) testing.

\section{EXPERIMENTAL RESULTS}

At the output of the HNLF-SPINE, the signal power is measured using an optical spectrum analyzer as the phase of the signal carrier $\mathrm{S}$ is swept relative to the phases of the pumps P1, P2 using the optical processor. Due to the inherent phase stability of this scheme, a precise dynamic characterization, i.e. with a QPSK modulated signal, of the full phase-sensitive response in the PSA stage is made possible. Static and dynamic characterizations are reported in Fig. 2 for a total input power of $28.6 \mathrm{dBm}$, showing a phase sensitive extinction ratio of $23.4 \mathrm{~dB}$ and $8.6 \mathrm{~dB}$ respectively. The phase sensitive response is clearly sinusodial with a period of $90^{\circ}$, indicating a stable phase relation between the carriers and the desired interaction between the $3 \phi$ and $-\phi$ idlers. Comparison between the input spectrum and output spectra for maximum amplification $\left(\phi=305^{\circ}\right)$ and deamplification $\left(\phi=350^{\circ}\right)$ are reported in Fig. 1(inset), showing the $8.6 \mathrm{~dB}$ difference between the generated idler power levels for the dynamic

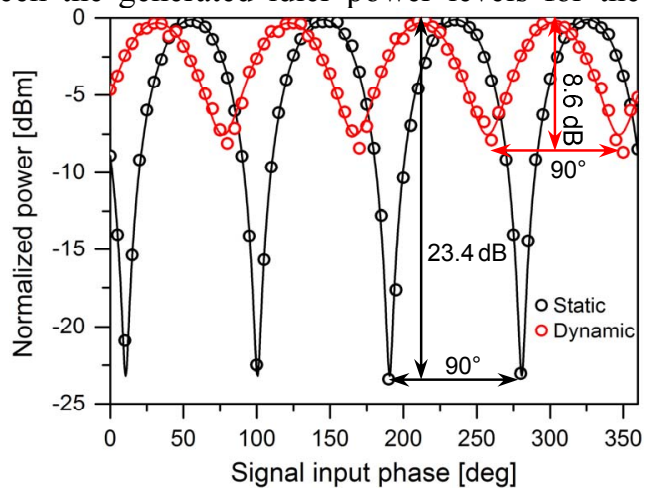

Fig. 2. Normalized phase sensitive gain of the signal as a function of signal phase with (red) or without (black) 10 Gbaud data modulation.

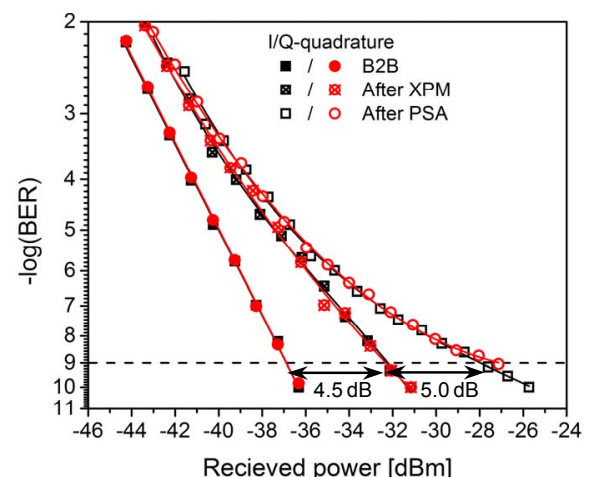

Fig. 3. BER curves B2B (solid), after XPM (crossed) and after PSA (open) for the I (black) and Q (red) quadrature components.

case. The significantly lower extinction ratio in the dynamic case is mainly caused by overlap between the FWM idlers, which limited the performance of the PSA.

The BER of the signal is measured back-to-back (B2B), as well as before and after the PSA stage (labelled After XPM and After PSA, respectively). The resulting BER curves are shown in Fig. 3. The QPSK modulation is indeed transferred to the locally generated carrier, and we are able to receive both the I and Q quadrature components errorfree after pre-conditioning. The modulation transfer (by DI and XPM) results in a power penalty of around $4.5 \mathrm{~dB}$ compared to the $\mathrm{B} 2 \mathrm{~B}$ reference. Insertion of the PSA stage results in the creation of a wavelength converted copy of the signal as desired for regeneration, although in these preliminary investigations we were uable to obtain the desired noise reduction. After PSA, both the I and Q components of the generated idler are received error-free, but with an added penalty of $\sim 5 \mathrm{~dB}$ at $\mathrm{BER}=10^{-9}$ due to a non-optimum amplifier configuration possibly combined with modulation due to pump saturation.

\section{CONCLUSION}

We have proposed and demonstrated a novel scheme for pre-conditioning of a QPSK data signal and generation of the required phase-locked pumps suitable for QPSK regeneration in a phase sensitive amplifier. The performance has been investigated for a 10-Gbaud QPSK signal and a power penalty of around $4.5 \mathrm{~dB}$ was observed at a BER of $10^{-9}$ for the signal pre-conditioning scheme. We propose this scheme could be used as a future QPSK regenerator, although this application has not been demonstrated in these preliminary investigations.

\section{ACKNOWLEDGMENT}

Danish Council for Independent Research (FTP) for funding the TOR project (grant no. 12-127224), and OFS Fitel Denmark ApS for providing the HNLF-SPINE.

\section{REFERENCES}

[1] R. Slavik et al., "All-optical phase and amplitude regenerator for next-generation telecommunications systems," Nature Photon. 4, 690 (2010).

[2] J. Kakande et al., "First demonstration of all-optical QPSK signal regeneration in a novel multi-format phase sensitive amplifier," Proc. ECOC'10, PD3.3 (2010)

[3] T. Richter et al., "QAM phase-regeneration in a phase-sensitive fiberamplifier," Proc. ECOC'13, We.3.A.2 (2013).

[4] N. K. Kjøller et al., "A Novel Phase-Locking-Free Phase Sensitive Amplifier based Regenerator,” Proc. OFC'15, W4C.2 (2015). 